\title{
Falsely Elevated Thyroid-Stimulating Hormone Results due to Interference by M-Component of IgG-Lambda Type
}

\author{
Anders Larsson $^{a} \quad$ Mats Gåfvels ${ }^{a}$ Torbjörn Karlsson ${ }^{b}$ \\ aSection of Clinical Chemistry, Department of Medical Sciences, Uppsala University, \\ Uppsala, Sweden; bSection of Haematology, Department of Medical Sciences, Uppsala \\ University, Uppsala, Sweden
}

\section{Keywords}

Erroneous laboratory test result - M-component - Myeloma - PEG- precipitation .

Thyroid-stimulating hormone

\begin{abstract}
Heterophilic antibodies but also M-components can interfere with laboratory tests causing erroneous results. We report the case of a 75 -year-old man with myeloma and a monoclonal immunoglobulin component (M-component) that caused elevated thyroid-stimulating hormone (TSH) results. The M-component was of the IgG-lambda type. Thyroid markers were analyzed repeatedly, and there was a clear association between IgG concentrations and TSH values $\left(R^{2}=0.724\right)$. The highest TSH value was $75 \mathrm{mIU} / \mathrm{L}$. Polyethylene glycol (PEG) precipitation of intact immunoglobulins was used to investigate if there was an antibody-related interference problem. The PEG treatment normalized the TSH value, showing that the cause of the elevated TSH result was due to interference caused by the M-component. In conclusion, it is important to remember that both heterophilic antibodies and $\mathrm{M}$-components may cause erroneous results.

(C) 2020 The Author(s).

Published by S. Karger AG, Basel
\end{abstract}

\section{Introduction}

The determination of thyroid-stimulating hormone (TSH) is one of our most frequently requested analyses. It is usually considered as a very reliable test to diagnose all common forms of hypo- and hyperthyroidism. Most TSH assays used clinically are sandwich immunoassays where one solid phase-bound antibody captures the TSH molecule and a second labeled 
Fig. 1. Association between IgG concentrations and TSH values from samples collected on the same date. The sample period was 2015-2019.

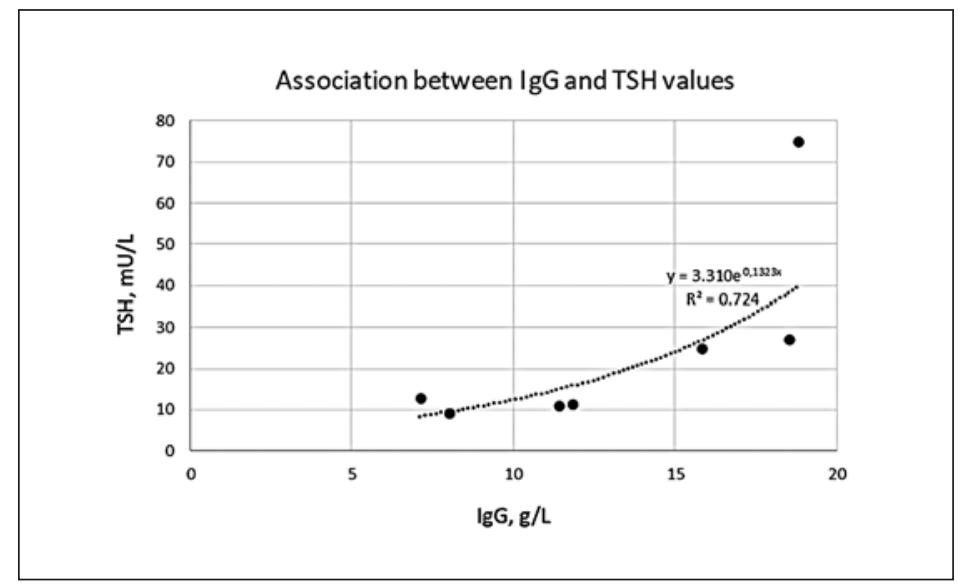

antibody is used to detect bound TSH. Antibodies present in the patient samples can form a bridge between the capture and detection antibody causing false positive results [1]. All immunoassays intended for clinical use include blocking agents, such as nonspecific IgG, from the same species as the assay antibodies to reduce the interference caused by heterophilic antibodies. Still, erroneous TSH results may occur in some patients. Although the presence of heterophilic antibodies is fairly common, there are very few reports on monoclonal immunoglobulin components (M-components) interfering in sandwich immunoassays [2, 3].

\section{Case Report}

We here report a 75-year-old man with an M-component that caused elevated TSH results. The patient has given his written informed consent to publish the case report. In 2015, he was diagnosed with an IgG-lambda myeloma. The myeloma produces both free lambda chain and intact IgG-lambda immunoglobulin. Immediately after diagnosis, bortezomib-based chemotherapy was initiated due to myeloma-related renal failure. The patient responded well clinically and biochemically to the therapy administered. He is currently in remission after 2 relapses requiring second- and third-line chemotherapy.

In April 2019, a serum sample from the patient was treated with polyethylene glycol (PEG) 6000 (12\% final concentration) to precipitate intact immunoglobulins. This is a standard test to remove heterophilic antibodies, but it will also remove intact M-components. The TSH value prior to the PEG precipitation was $9.8 \mathrm{mIU} / \mathrm{L}$ and $0.89 \mathrm{mIU} / \mathrm{L}$ after the serum was treated with PEG. The free T4 value was $18.7 \mathrm{pmol} / \mathrm{L}$ (reference interval 12-22) and free T3 was $4.1 \mathrm{pmol} / \mathrm{L}$ (reference interval 3.1-6.8). At the same time, the patient had a total IgG value of $8 \mathrm{~g} / \mathrm{L}$, with the M-component being the dominating part of the IgG, IgA $0.12 \mathrm{~g} / \mathrm{L}$, IgM $<0.05 \mathrm{~g} / \mathrm{L}$, and free lambda chain of $200 \mathrm{mg} / \mathrm{L}$, while the free kappa chain was $<1 \mathrm{mg} / \mathrm{L}$. The patient also had a creatinine at $136 \mu \mathrm{mol} / \mathrm{L}$ (reference 60-105), creatinine-estimated GFR 40 $\mathrm{mL} / \mathrm{min} / 1.73 \mathrm{~m}^{2}$ (reference 50-90), hemoglobin $146 \mathrm{~g} / \mathrm{L}$ (reference 130-170), platelets 104 $\times 10^{9} /$ L (reference 150-350), red blood cells $4.35 \times 10^{12} /$ L (reference $4.3-5.7$ ), and white blood cells $6.6 \times 10^{9} / \mathrm{L}$ (reference 3.5-9.0). Since 2015, the IgG levels had varied between 3.9 and $18.8 \mathrm{~g} / \mathrm{L}$ and the TSH had varied between 9.3 and $75 \mathrm{mIU} / \mathrm{L}$. For TSH and IgG ordered at the same date, there was a clear association between the IgG and TSH values $\left(y=3.310 \mathrm{e}^{0.1323} x\right.$; $R^{2}=0.724$; Fig. 1). TSH, free T4, and free T3 were analyzed on a Cobas E602 (Roche Diagnostics Scandinavia, Solna, Sweden) and IgG, IgA, and IgM were analyzed on an Architect 16000. 


\section{Discussion/Conclusion}

It is known that M-components/paraproteins can interfere with laboratory tests, such as bilirubin, calcium, creatinine, C-reactive protein, phosphate, and T3 and T4 [4]. There are very few reports on interference of paraproteins in TSH assays. Ross et al. [1] reported a case of interference by a M-component of the IgM type, and Imperiali et al. [5] reported a case of interference by an IgG/IgM M-component. We here report the interference in a TSH assay by an IgG M-component. We also show that PEG precipitation can be used to verify interference problems due to M-components.

In conclusion, it is important to be aware of interference problems when evaluating test results from patients with paraproteins. The use of PEG precipitation to remove the $\mathrm{M}$-component prior to TSH testing is an effective method to verify suspected interference problems.

\section{Statement of Ethics}

The study was conducted in accordance with the Declaration of Helsinki. The patient has given his written informed consent to the submission and publishing of the content of the case report.

\section{Disclosure Statement}

The authors have no conflicts of interest to declare.

\section{Funding Sources}

The Uppsala University Hospital Research Fund, Sweden, supported this study.

\section{Author Contributions}

T.K. collected the clinical data, while A.L. and M.G. were responsible for the laboratory work. A.L. drafted the initial manuscript. All authors read and approved the final version.

\section{References}

1 Ross HA, Menheere PP, Thomas CM, Thomas CM, Mudde AH, Kouwenberg M, et al. Interference from heterophilic antibodies in seven current TSH assays. Ann Clin Biochem. 2008;45(Pt 6):616-8.

2 Dalal BI, Brigden ML. Factitious biochemical measurements resulting from hematologic conditions. Am J Clin Pathol. 2009;131(2):195-204.

3 Kricka LJ. Human anti-animal antibody interferences in immunological assays. Clin Chem. 1999;45(7):94256.

4 Cissewski K, Faix JD, Reinwein D, Moses AC. Factitious hyperthyroxinemia due to a monoclonal IgA in a case of multiple myeloma. Clin Chem. 1993;39(8):1739-42.

5 Imperiali M, Jelmini P, Ferraro B, Keller F, della Bruna R, Balerna M, et al. Interference in thyroid-stimulating hormone determination. Eur J Clin Invest. 2010;40(8):756-8. 